\title{
A COMPUTATIONAL APPROACH TO DUCTILE CRACK GROWTH UNDER LARGE SCALE YIELDING CONDITIONS
}

\author{
LIN XIA $\uparrow$ C. FONG SHIH $\dagger$ and JOHN W. HUTCHINSON $\ddagger$ \\ †Division of Engineering, Brown University, Providence, RI 02912, U.S.A. and $\ddagger$ Division of Applied \\ Sciences, Harvard University, Cambridge, MA 02138, U.S.A.
}

(Received 22 June 1994 ; in revised form 6 October 1994)

\begin{abstract}
Mode I crack initiation and growth under plane strain conditions in tough metals is computed using an elastic-plastic continuum model which accounts for void growth and coaleseence ahcad of the crack tip. The material parameters are the Young's modulus, yield stress and strain hardening exponent of the metal, along with the parameters characterizing the spacing and volume fraction of voids in material elements lying in the plane of the crack. For a given set of these parameters and a specific specimen, or component, subject to a specific loading, relationships among load, load-line displacement and crack advance can be computed with no restrictions on the extent of plastic deformation. Similarly, there is no limit on crack advance, except that it must take place on the symmetry plane ahead of the initial crack. Suitably defined measures of crack tip loading intensity, such as those based on the $J$-integral, can also be computed, thereby directly generating crack growth resistance curves. In this paper, the model is applied to five specimen geometries which are known to give rise to significantly different crack tip constraints and crack growth resistance behaviors. Computed results are compared with sets of experimental data for two tough steels for four of the specimen types. Details of the load, displacement and crack growth histories are accurately reproduced, even when extensive crack growth takes place under conditions of fully plastic yielding.
\end{abstract}

\section{INTRODUCTION}

Two main obstacles to the development of nonlinear fracture mechanics for application to tough, ductile metals have come sharply into focus since the 1970s when the subject first began to receive appreciable attention. A strong geometry dependence of crack growth resistance emerged which is associated with different levels of crack tip constraint under large scale yielding conditions. The early theoretical work of McClintock (1971) clearly suggested that such a dependence should be expected, and the recent systematic experimental studies of Hancock et al. (1993) provide the most definitive data available to date on crack tip constraint effects. The other aspect of nonlinear fracture mechanics which has proved difficult for all predictive approaches is extensive crack growth. At a fixed level of crack tip constraint, there are sound arguments which can be used to justify an approach to analyzing crack growth and stability using crack resistance data based on the $J$-integral, as long as the amount of crack growth is sufficiently small. As a practical matter, this approach has been called 


\section{Fracture Test Specimens}

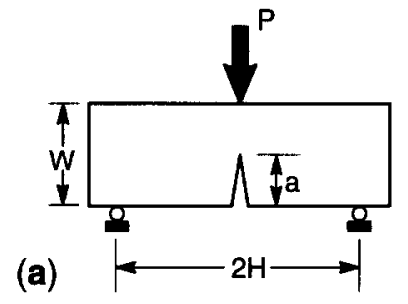

(b)
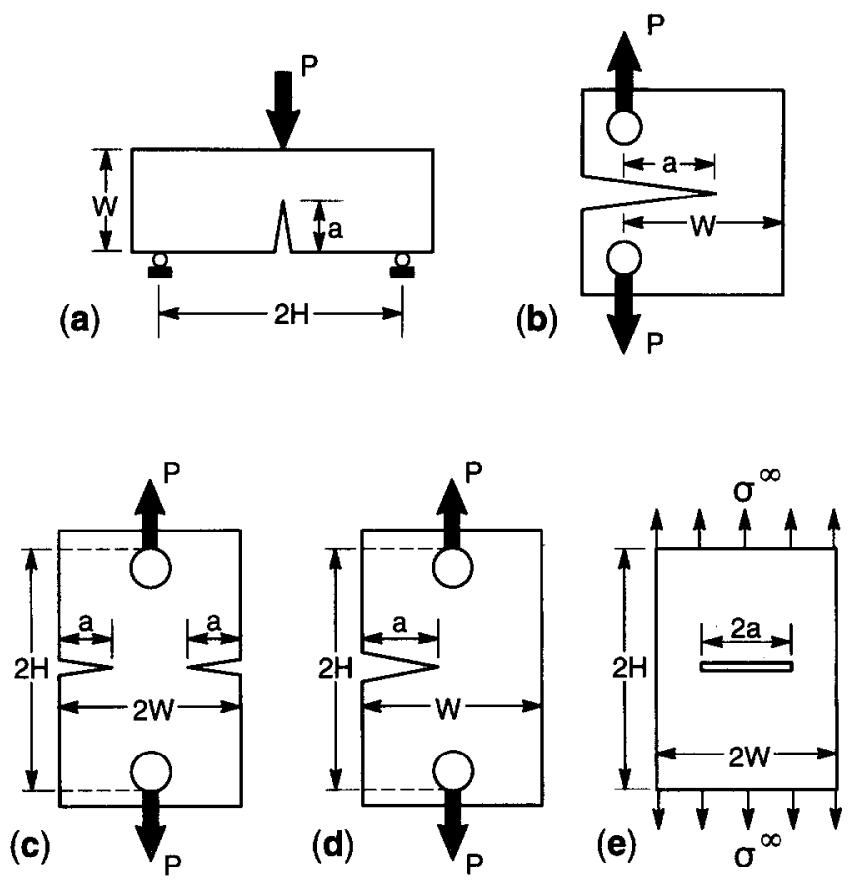

Fig. 1. Crack geometries : (a) three point bend (TPB) ; (b) compact tension specimen (CT); (c) double edgenotched tension strip (DENT); (d) single edge-notched tension strip (SENT); and (e) center-cracked panel (CCP).

into question for several important applications involving crack extensions which appear to exceed justifiable limits.

In this paper a computational model for mode I plane strain crack growth in tough, ductile metal alloys is applied to predict crack growth and load-displacement behavior for the five specimen geometries shown in Fig. 1. Extensive comparisons with experimental data will be made for four of the five specimen types for two steels tested by Joyce and Hackett (1991) and Joyce and Link (1994). Each application involves both large scale yielding and reasonably large amounts of crack growth. The model employs cell elements on the plane ahead of the crack, each of which contains a void. The void-containing elements are embedded within a conventional elastic plastic continuum whose properties coincide with those of the metal. Under increasing load, the voids grow and coalesce to form new crack surface thereby advancing the crack. In principle, there is no limit to the extent of crack growth that can be considered, as long as it occurs on the original crack plane. The present paper is the third in a sequence which started with the introduction of the model and its first application to small scale yielding behavior by Shih and Xia (1995) and was followed by a discussion 
of computational details by Xia and Shih (1995). The primary aim of the present paper is to demonstrate convincingly by way of examples that this computational model, and others of its type, can accurately predict the full details of load-displacement and crack growth behavior in both test specimens and cracked structural components under large scale yielding conditions.

The history of the development of computational models for crack growth in ductile solids has not been one of continuous progress. Early progress in developing computational models for predicting crack growth in ductile matcrials was achicved in work representative of that of Kanninen et al. (1979) and Shih et al. (1979). These workers used a local criterion, or combination of criteria, for crack advance, such as attainment of a critical crack tip opening angle, which, in turn, was chosen such that the computational model gave as good a fit as possible to one set of crack growth data for the material. Once the criterion was established, the model could be applied to other applications involving the same material. Rice and coworkers (e.g. Drugan et al., 1982) carried out a fundamental investigation of near-tip fields for mode I propagation of plane strain cracks. They demonstrated that a criterion based on maintenance of an invariant crack tip opening profile was capable of reproducing behavior once crack propagation was underway in compact tension specimens of a particular class of steels under moderate to large scale yielding conditions (Hermann and Rice, 1980). In spite of the promise evident in this early work, there followed a hiatus in which rather little further effort was expended on computational aspects of mode I plane strain crack growth in ductile metals. In retrospect, one limitation of the early approaches was the inability of the local fracture criteria to capture the strong dependence on crack tip constraint. Nevertheless, it seems fair to say that the main elements of a computational model were in place some years ago, and substantial progress would have been likely had the subject been actively pursued.

More recent efforts in this area have focused on the development of models which, like the present model, incorporate void growth as a fracture process in the computational scheme. Tvergaard and Needleman (1984) demonstrated the power of such models when applied to predict the development of the cup-cone mode of fracture in necking of a round tensile bar. Their continuum model employed the Gurson (1977) constitutive relation for an elastic-plastic solid containing voids. Subsequently, Needleman and Tvergaard (1987) extended McMeeking's (1977) study of the initiation of crack growth. They focused on the interaction between the void nearest the crack tip and the tip itself by treating large voids as discrete entities and by modeling a second population of much smaller voids using the Gurson relation. In work which is closest in spirit to that developed here, Sun and Schmitt (1990) and Brocks et al. (1995) have adopted a version of the computational model proposed by Needleman and Tvergaard and have applied the model to analyze fully plastic cracking behavior of tough steels for various specimen geometries. Work based on a local failure criterion was originated and pursued independently by Rousselier (1987) and coworkers, who developed their own mechanics model for void damage as an alternative to the Gurson model. Parameters specifying the initial void population are chosen such that the model reproduces the experimental behavior of a given metal tested using notched tensile specimens. In this way, the model is calibrated so that it is capable of reproducing behavior under high constraint conditions. The dependence of crack growth 
resistance on constraint, as tied to specimen geometry, has been demonstrated (Rousselier et al., 1989b). A number of important applications of this approach have been made to predict fracture of specimens and components of ductile metals, including those reported by Mudry et al. (1989), Rousselier et al. (1989a), Bethmont et al. (1990), Bilby et al. (1993), and Li et al. (1993).

Independently of the plane strain studies, work along parallel lines has been pursued using local crack tip criteria for mode I in plane stress for application to crack growth in thin shect materials by various workers, including Hellmann and Schwalbe (1984), Budiansky and Sumner (1985) and Newman et al. (1993). In some respects, plasticity effects are less complicated in thin sheets because constraint effects appear to be less important. Under some circumstances, plastic deformation can be modeled effectively using a plastic zone concentrated on the line ahead of the crack. The recent work of Newman et al. (1993) has carried the computational approach the furthest with application to the burst capacity of an aircraft fuselage having a major crack in combination with small crack damage at rivets in the lap joint. This application illustrates the potential of a computational approach to crack growth and stability to deal with complicated structural problems.

The present computational model is introduced and discussed in the following section. In Section 3, a brief study of the dependence of the predictions of the model on the main parameters is presented. The main applications of the model are made in Section 4 where theoretical and experimental results are given for four specimen types made of the pressure vessel steel, A533B. Other comparisons are made for an even tougher class of steels, A710. Once the material parameters of the model have been specified, the approach permits computation of relationships among loads, displacements and crack growth, including states where stability is lost. A crack growth resistance curve no longer plays the central role in this approach. Nevertheless, because there is such a long experience using resistance curves, we have calculated resistance curves for each of the specimens and materials in the form of $J$ versus crack advance, $\Delta a$. These are compared with the corresponding experimentally measured resistance curves. One advantage of the computational model is that it permits one to compute various contending crack tip parameters. We have used this advantage to compare computed values of the $J$-integral on contours remote from the crack tip with formulas that are used to estimate $J$ from experimental load-displacement records, such as the ASTM (1993) procedure and the modified $J$-measure suggested by Ernst (1989). It is important to keep in mind, however, that issues surrounding material crack growth resistance and its measurement can be relegated to a secondary role in the present approach.

The present study, when taken together with the prior work based on the local fracture approach of Rousselier and coworkers and the work of Sun and coworkers based on Needleman and Tvergaard's Gurson theory model, provides a fairly extensive body of results against which the predictive power of this type of computational approach can be weighed. There are some differences between the approaches in terms of the number and choice of damage parameters and in the way in which these parameters are to be chosen. Nevertheless, we believe that a convincing case for the predictive power for this type of approach now exists and that its potential as a tool for analyzing ductile fracture is evident. 

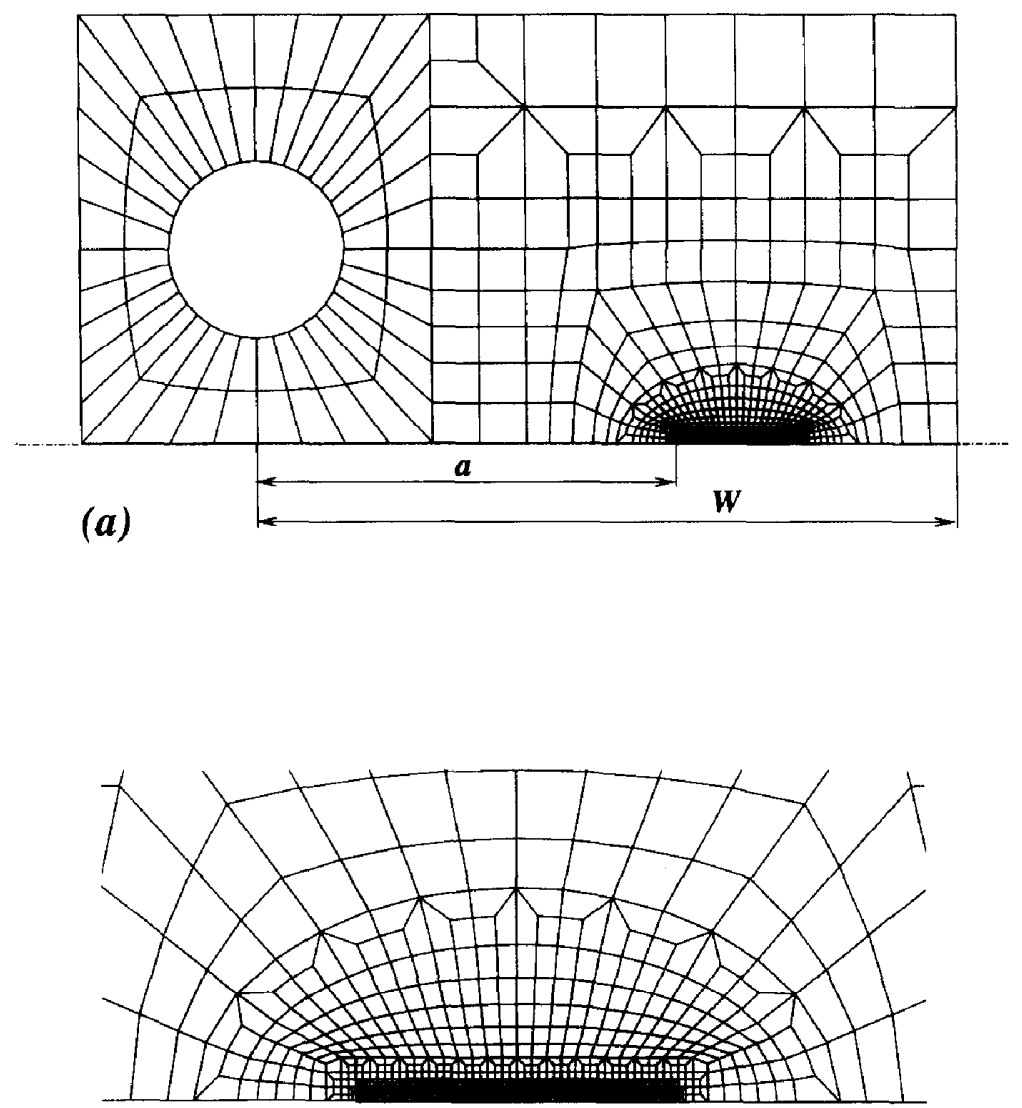

(b)

Fig. 2. Geometry and finite element mesh for the compact tension specimen. (a) Upper half of the specimen; and (b) blow-up of the refined mesh ahead of the initial crack tip.

\section{THE COMPUTATIONAL MODEL}

Geometric details of the model are illustrated for the compact tension specimen in Figs 2 and 3. The deformation is taken to be plane strain and, apart from the voidcontaining elements on the line directly ahead of the crack, the material is represented by the standard $J_{2}$ flow theory of plasticity. With $E$ as the Young's modulus, $v$ as Poisson's ratio and $\sigma_{0}$ as the initial tensile yield stress, the true stress-logarithmic strain curve is taken to be

$$
\begin{array}{ll}
\varepsilon=\frac{\sigma}{E} & \sigma<\sigma_{0}, \\
\varepsilon=\frac{\sigma_{0}}{E}\left(\frac{\sigma}{\sigma_{0}}\right)^{1 / N} & \sigma \geqslant \sigma_{0},
\end{array}
$$

where $N$ is the strain hardening exponent. A finite strain formulation of the elastic- 

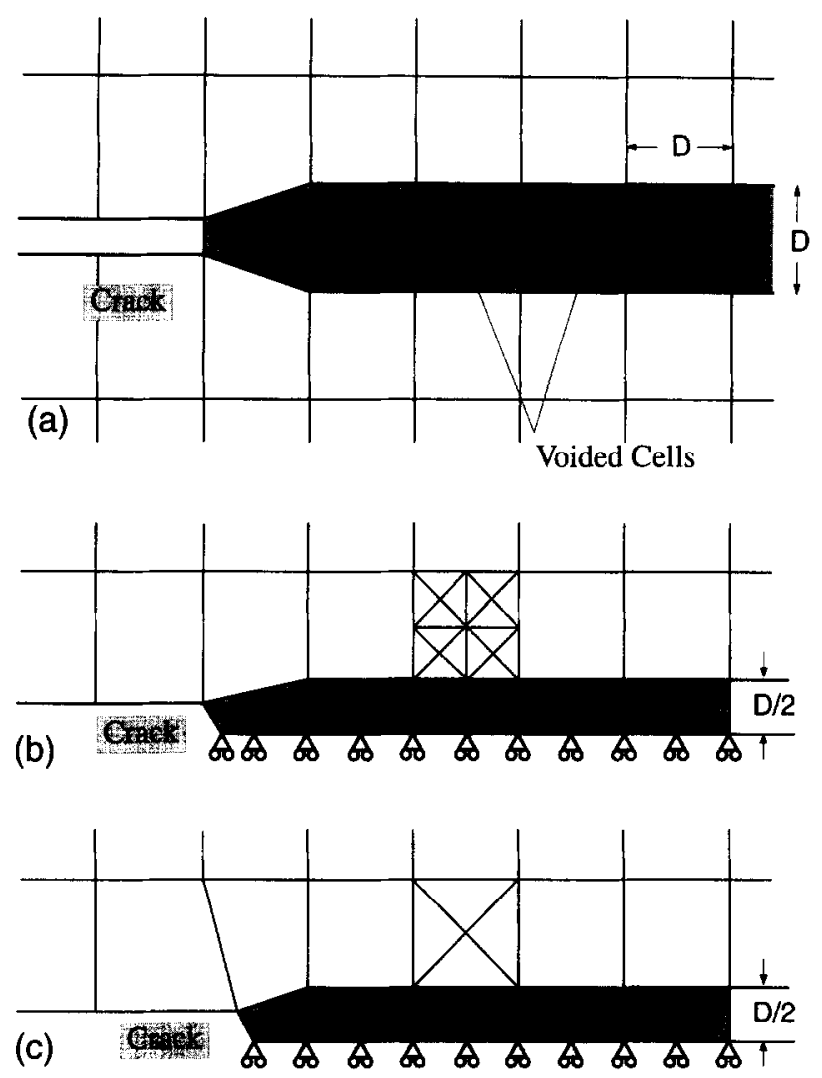

Fig. 3. (a) Schematic of void-containing elements in slab along the projected crack line; (b) sub-element Scheme A used in computing most of the results in the paper; and (c) sub-element Scheme B.

plastic continuum is employed, although finite strain effects are not dominant in determining the response of any of the geometries analyzed. A finite element representation of the elastic-plastic continuum is employed based on quadrilateral elements comprised of four constant strain triangular elements. The mesh used in the analysis of the compact tension geometry is shown in Fig. 2 where it can be seen that a highly refined rectilinear mesh stretches out on the ligament ahead of the tip where the crack growth will occur. Various refinements of the mesh have been used. Most calculations reported in this paper used on the order of 1000 elements, with roughly 100 elements distributed on the ligament ahead of the crack where crack advance takes place.

Directly ahead of the initial crack tip in the plane of fracture is placed a single slab of fracture process elements containing voids, as depicted in Fig. 3. Viewed as threedimensional entities, the elements are initially cubes with dimension $D$ on a side. With the symmetry associated with mode I, only the upper half of the elements will be considered. In plane strain, the cube-elements are subdivided into constant strain triangular sub-elements according to several schemes. The bulk of the results presented here have been computed using Scheme A shown in Fig. 3(b), but some results 
obtained using Scheme B in Fig. 3(c) will also be presented to illustrate the sensitivity of the predictions to the choice of scheme. Each triangular sub-element shaded in Fig. 3 contains a single spherical void with initial volume fraction $f_{0}$, such that $f_{0}$ represents the initial volume fraction of the voids in the slab. Thus, in addition to the material parameters already identified (i.e. $E, v, \sigma_{0}$ and $N$ ), the material slab introduced on the fracture plane is characterized by the initial void volume fraction $f_{0}$ and the initial slab height $D$, with an implicit dependence on the choice of sub-element scheme. The elements in the slab, likc those in the remainder of the body, are forced to undergo plane strain deformations. While a $2 \mathrm{D}$ plane strain formulation is used in the present study, the constitutive behavior of the void-containing elements derives from a $3 \mathrm{D}$ model with $f_{0}$ as the initial volume fraction of spherical voids.

The multi-axial stress-strain response of the slab elements is represented by Gurson's (1977) stress-strain relation for an elastic-plastic solid containing voids. Gurson's relation was proposed for situations where the characteristic length of the deformation field is long compared to the void spacing, and, consequently, the void volume fraction was to be regarded as being averaged over many voids. But, in fact, the derivation of Gurson's constitutive relation is based on an analysis of a single cell containing a centered spherical void whose initial volume fraction of the cell is $f_{0}$. Although it was not originally intended for this purpose, the Gurson relation can be used to characterize the stress-strain behavior of a single void-containing cell element. At the heart of Gurson's relation is the yield condition

$$
\Phi\left(\sigma_{\mathrm{e}}, \sigma_{\mathrm{m}}\right)=\left(\frac{\sigma_{\mathrm{e}}}{\bar{\sigma}}\right)^{2}+2 q_{1} f \cosh \left(\frac{3 q_{2} \sigma_{\mathrm{m}}}{2 \bar{\sigma}}\right)-\left[1+\left(q_{1} f\right)^{2}\right]=0 .
$$

Here $\sigma_{\mathrm{c}}$ is the effective Mises stress formed using the Cauchy stress, $\sigma_{\mathrm{m}}$ is the mean of the Cauchy stress, $\bar{\sigma}$ is the current flow stress, $f$ is the current void volume in the $3 \mathrm{D}$ sense mentioned above, and $q_{1}$ and $q_{2}$ are the Tvergaard (1990) factors introduced to improve the accuracy of the model $\left(q_{1}=1.25\right.$ and $q_{2}=1.0$ in the calculations reported below). For a cell element at yield, the plastic strain increment is related to the Jaumann rate of the Cauchy stress $\dot{\sigma}_{i j}$ by

$$
\dot{\varepsilon}_{i j}^{\mathrm{p}}=\frac{1}{h} \frac{\partial \Phi}{\partial \sigma_{i j}} \frac{\partial \Phi}{\partial \sigma_{k l}} \dot{\sigma}_{k l},
$$

where $h$ depends on the current hardness level through the stress-strain relation (1) and the void volume fraction as specified in full detail by Gurson (1977) or in the review article by Tvergaard (1990). The void-free limit with $f_{0}=0$, is precisely the $J_{2}$ flow theory solid characterizing the material off the fracture plane. Given an initial void volume fraction $f_{0}$ and the stress-strain relation of the base material (1), the history of $f$ as a function of the stress or strain is computed using the constitutive model. Under increasing strain, the void volume fraction in a given cell reaches some level where the cell no longer supports any traction across the plane of the crack and the crack advances across that cell. The Gurson model does not adequately supply the traction relation during the final phase prior to material separation. In the present work, the Gurson relation is used until the value $f=f_{\mathrm{E}}=0.2$ is reached, at which point the cell is assumed to cease to support traction. When a cell attains $f=f_{\mathrm{E}}$, it is 
rendered extinct by the element vanish technique of Tvergaard (1982), which reduces the traction carried by the element to zero in a 20 -increment release step.

In summary, the material properties off the fracture plane are $E, v, \sigma_{0}$ and $N$. These same parameters characterize the slab of material along the fracture plane along with the two additional damage parameters, the initial slab height $D$ and the initial volume fraction $f_{0}$. Given a choice of sub-element scheme, as further discussed below, the model becomes complete. For a given geometry with an initial crack subject to either a monotonically increased load or load-line displacement, the history of crack advance can be computed along with the history of any other load or displacement quantities of interest. A number of examples will be given in the paper. In applying the model to a specific material the two parameters, $D$ and $f_{0}$, will be chosen such that the model gives a best fit to one set of experimental crack growth data for that material. These parameters are not chosen to replicate microscopic observations of void spacing and initial void volume fraction. Their values will depend somewhat on the choice of subelement scheme. In this sense, the model should be regarded as phenomenological, but with a microstructural basis. Additional microstructural features could be incorporated, including parameters characterizing stress or strain levels at which voids are nucleated, as has also been considered by Gurson [see Tvergaard's (1990) review]. At some later date it may prove worthwhile to embellish the model with additional parameters, as Brocks et al. (1995) have done, but the present study suggests that this may not be necessary.

\subsection{Sub-element Scheme A}

This scheme uses the arrangement of triangular void-elements shown in Fig. 3(b). The top half of the square of dimension $D / 2$ by $D$ is subdivided into eight constant strain triangular sub-elements, each of which contains a void with initial volume fraction $f_{0}$. Various conventions for identifying the effective location of the crack tip are possible. For each of the two schemes here, we follow Becker et al. (1989) and advance the crack tip when the void volume fraction averaged over a set of subelements reaches 0.1 . For Scheme A, the average $\bar{f}$ is taken over the four sub-elements comprising one small square of dimension $D / 2$ by $D / 2$. Thus, the crack tip is advanced by $D / 2$ across this four-element square when $\bar{f}$ reaches 0.1 . This is a somewhat arbitrary choice which is decoupled from the point of complete loss of load carrying capacity of the sub-clements. As already mentioned, a sub-element continues to carry load until $f=f_{\mathrm{E}}=0.2$, and then the traction it carries is reduced to zero in 20 steps.

\subsection{Sub-element Scheme B}

The arrangement in this scheme, which is shown in Fig. 3(c), employs only three sub-elements in the half-square of dimension $D / 2$ by $D$. As in Scheme $A$, the crack is advanced when $\bar{f}=0.1$. Now, however, the average is taken over the three elements making up the half-square, and the crack advances a distance $D$. Two versions of the element vanish procedure were used. The first was identical to that in Scheme A with the traction on each sub-element reduced to zero in 20 steps when $f=f_{\mathrm{E}}$ in that subelement. In the second version, an average value of the void volume fraction $\bar{f}$ was taken over the same three sub-elements used for advancing the location of the crack 
tip. When this average reached $f_{\mathrm{E}}=0.2$ the tractions carried by all three sub-elements were simultaneously reduced to zero in 20 steps.

Most of the computations reported in this paper were carried out using Scheme A. A limited number of results were computed using the two variations of Scheme B as well, with the purpose of establishing the sensitivity of the choice of sub-element scheme on the model predictions. These are reported in the next section. There is also a sensitivity of both schemes to the way in which the crack tip location is defined and to the value assigned to $f_{\mathrm{E}}$. The dependence of the predictions on these two aspects is relatively weak and will not be illustrated here.

\section{PARAMETRIC, DFPENDENCIFS}

The present approach makes contact with a model of mode I plane strain crack growth developed by Tvergaard and Hutchinson $(1992,1994)$ which represents the fracture process zone by a traction-separation relation embedded within the elasticplastic continuum. Consider imposition of a uniform separation $\delta$ of the upper and lower faces of the void-containing slab, subject to zero average strain in the two directions parallel to the slab. For such uniaxial straining, the traction-separation relations computed for Schemes A and B are identical, given the same tensile stressstrain data (1) and the same values of $D$ and $f_{0}$. Moreover, they are identical to those presented in some detail by Tvergaard and Hutchinson (1992, Section 4) who used the Gurson model to generate traction-separation relations for a slab containing voids. The condition of zero straining parallel to the slab (i.e. uniaxial straining perpendicular to the slab) models the highest constraint to be expected. The work of separation per unit area under these conditions is $\Gamma_{0}=\int \sigma \mathrm{d} \delta$, which was shown by Tvergaard and Hutchinson to be approximately $\sigma_{0} D / 2$, with only a weak dependence on $N$ and $f_{0}$. The significance of $\Gamma_{0}$ will emerge below.

It is useful to contemplate application of the present model to crack growth under small scale yielding conditions such that the crack length and other length parameters characterizing the specimen geometry enter only collectively through $J$. With $K$ as the applied stress intensity factor characterizing the remote field and with $J=\left(1-v^{2}\right) K^{2} / E$, dimensional considerations dictate that the computed history of $J$ versus $\Delta a$ must have the general form

$$
J=\sigma_{0} D F\left[\frac{\Delta a}{D}, f_{0}, N, \frac{\sigma_{0}}{E}\right]
$$

where $F$ is a dimensionless function of the variables shown. There is also an implicit dependence on the choice of sub-element scheme and on other variables such as $v$ and $f_{\mathrm{E}}$. In small scale yielding, both the ordinate and abscissa of the resistance curve necessarily scale with $D$.

This relation can be cast in a slightly different form which further elucidates the parametric dependencies. Following Tvergaard and Hutchinson (1992), define a reference plastic zone size by 


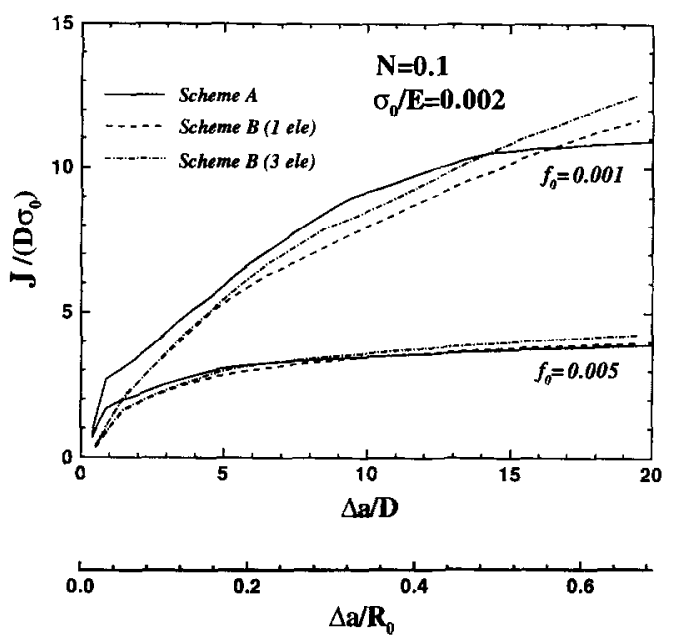

Fig. 4. Sensitivity of the resistance curves in small scale yielding to sub-element schemes used to represent the void-containing slab ahead of the crack. The results of $f_{0}=0.005$ are representative of the steel A533B and those for $f_{0}=0.001$ are representative of $A 710$. The designation 3 "ele" refers to the version of Scheme $B$ where the void volume fraction is averaged over 3 sub-elements in determining when the traction is to be reduced to zero; 1 "ele" refers to the other version based on an element by element release.

$$
R_{0}=\frac{1}{3 \pi\left(1-v^{2}\right)} \frac{E \Gamma_{0}}{\sigma_{0}^{2}} \cong \frac{1}{6 \pi\left(1-v^{2}\right)} \frac{E}{\sigma_{0}} D .
$$

This is the standard estimate of the plane strain zone size for small scale yielding with $J$ identified with the work of the fracture process, $\Gamma_{0} \approx \sigma_{0} D / 2$. It serves as a meaningful length quantity with which to normalize the crack advance in small scale yielding. Thus, an equivalent alternative to (4) is

$$
J=\Gamma_{0} \widetilde{F}\left[\frac{\Delta a}{R_{0}}, f_{0}, N, \frac{\sigma_{0}}{E}\right],
$$

which emphasizes the special role of $\Gamma_{0}$. Although less apparent, it still follows that the $J$-resistance curve scales with $D$, because both $\Gamma_{0}$ and $R_{0}$ are proportional to $D$.

Resistance curves in the form of $J /\left(D \sigma_{0}\right)$ as a function of $\Delta a / D$ computed for the present model in small scale yielding have been given in the earlier paper by Shih and Xia (1995) displaying the effect of different choices of $N$ and $f_{0}$. Two examples are shown here in Fig. 4 for $N=0.1, \sigma_{0} / E=0.002$, and $f_{0}=0.001$ and 0.005 . As will be seen later, the set of parameters with $f_{0}=0.005$ is representative of the steel A533B, while that with $f_{0}=0.001$ approximately characterizes $\mathrm{A} 710$. Three curves are shown for each case, one for sub-element Scheme A and one for each of the two versions of Scheme B. The abscissa is shown with two scales, $\Delta a / D$ and $\Delta a / R_{0}$, connected by (5).

The difference between the two procedures in Scheme B for reducing the tractions to zero is small. For both choices of $f_{0}$ there is significant sensitivity evident in Fig. 4 to the choice of sub-element scheme within the first several crack advance steps for $\Delta a$ less than about $2 D$. For the case $f_{0}=0.005$, there is little discrepancy among three predictions for crack advances beyond $2 D$. For the material with the higher tearing 
resistance $\left(f_{0}=0.001\right)$, there is a substantial intermediate range of growth where the differences among the three computed resistance curves are relatively small. At values of $\Delta a$ greater than $15 D$ a clear difference between the predictions of Schemes $A$ and $\mathrm{B}$ emerges, with $\mathrm{A}$ indicating the attainment of steady-state conditions, while the resistance continues to increase according to $\mathrm{B}$. An argument for why the material with very high tearing resistance shows more sensitivity to the choice of sub-element scheme than that with $f_{0}=0.005$ is as follows. Tvergaard and Hutchinson (1992) showed that the ratio of the extent of the fracture process zone ahead of the tip to the void spacing diminishes as $f_{0}$ becomes smaller. When $f_{0}$ is relatively large the ratio is large compared to unity and the fracture process zone extends over multiple voids. However, for values of $f_{0}$ as small as 0.001 , this ratio is on the order of unity implying that the fracture process is dominated by the interaction of the tip and the nearest void to it. Thus, it is not surprising that there is some sensitivity for this case to details of the arrangement of the sub-elements in the slab containing the voids.

The differences between schemes seen in Fig. 4 arise when the same values of $D$ and $f_{0}$ are used in each scheme. In applications of the model to a specific material, such as those made later in Section 4, it is recommended that one first choose the subelement scheme and then, secondly, pick $D$ and $f_{0}$ to best reproduce one set of experimental data for the material. In the same way, one could attempt to adjust the values of $D$ and $f_{0}$ for one of the schemes in Fig. 4 relative to those of the other such that the curves for the two schemes are brought into even better correspondence. We leave for subsequent work such issues related to the choice of sub-element scheme and as to whether other damage parameters, such as a void nucleation stress or strain, are needed to reproduce crack growth resistance better for certain materials. In all the applications reported below, Scheme A has been used. It is also worth remarking at this point that the initiation of crack growth is not a primary event for the two tough steels considered in this paper. Thus, the sensitivity of the very early crack growth resistance to the scheme seen in Fig. 4 is not particularly significant.

Now consider the three point bend geometry (TPB) of Fig. 1(a). Specifically, consider a specimen with $W=50 \mathrm{~mm}$ and initial crack length $a=0.6 W$. Fix the following material properties: $\sigma_{0}=400 \mathrm{MPa}, E / \sigma_{0}=500, \nu=0.3$ and $D=200 \mu \mathrm{m}$. The effects of varying the strain hardening exponent, $N$, and the initial void volume fraction, $f_{0}$, computed using sub-element Scheme A are displayed in Figs 5 and 6. The choice of parameters results in crack growth behavior which occurs under fully plastic conditions for all but the two larger values of $f_{0}$, for which crack growth initiates under large scale yielding.

Figure 5(a) shows predictions of the model computed with $f_{v}=0.005$ and three values of $N$ in terms of load per unit thickness, $P$, plotted against the load-line displacement $\Delta$. The companion curves of $J$ as a function of crack advance $\Delta a$ are shown in Fig. 5(b). It is evident that the behavior of the specimen depends strongly on the strain hardening parameter $N$. The crack grows more than a quarter of the way across the initial ligament in these examples. The $J$-values in Fig. 5(b) fand in Fig. 6(b)] were computed using the $J$-integral on a contour remote from the tip of the crack. Values of the $J$-integral on remote contours are found to be nearly pathindependent and within $5 \%$ of the values computed from the numerical results for load and crack advance versus load-line displacement using the so-called $\eta$-factor 

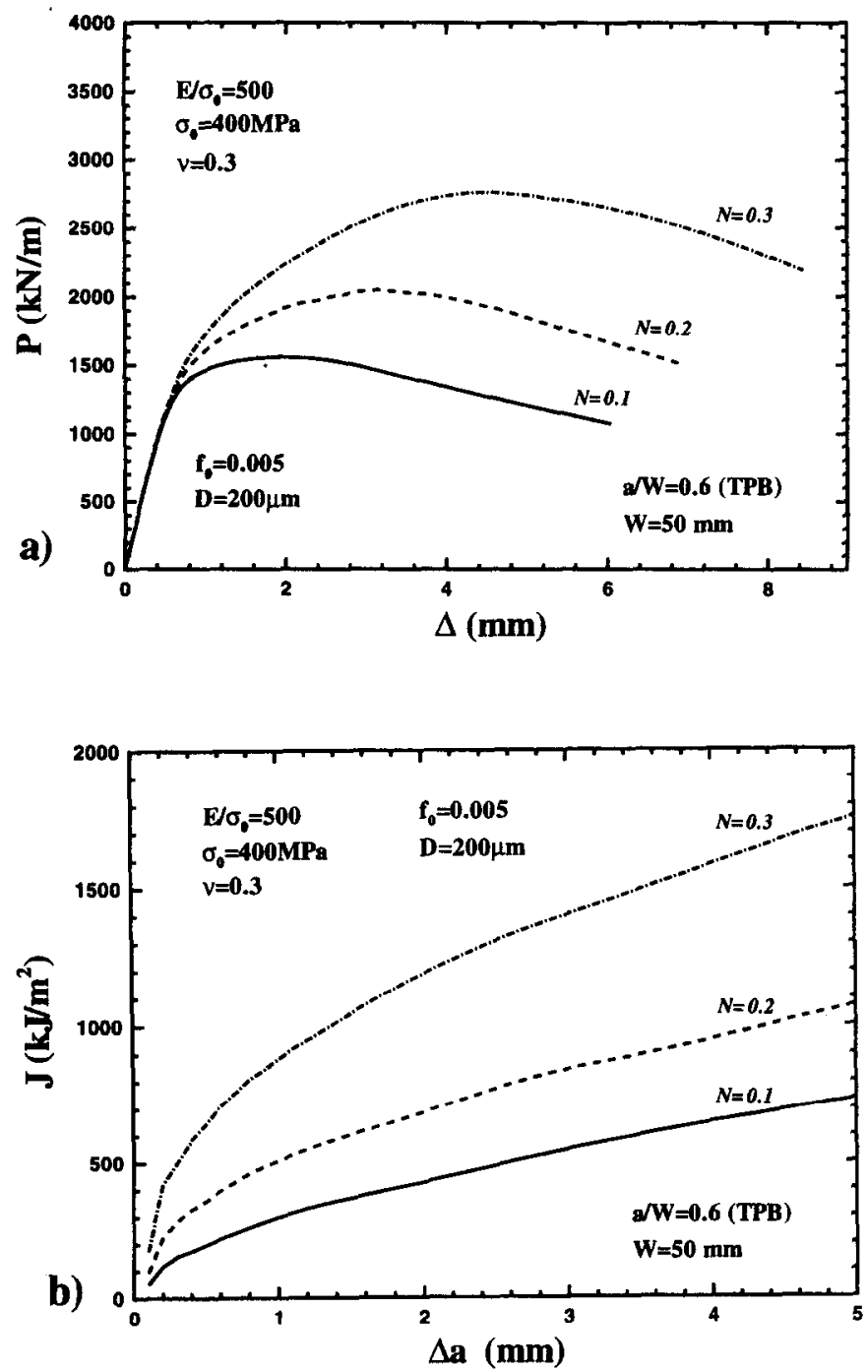

Fig. 5. Effect of variations in $N$ on the load-displacement relation in (a) and on the relation between $J$ and crack advance in (b). Three point bend geometry with $a / W=0.6$ and $W=50 \mathrm{~mm}$.

method recommended by ASTM (1993). Some issues surrounding the choice of an extended definition of $J$ under large scale yielding with extensive crack growth will be taken up in the last section.

The corresponding role of the initial void volume fraction $f_{0}$ is seen in Fig. 6 for $N=0.1$. A factor of 10 reduction in initial void volume fraction, from 0.01 to 0.001 , has a significant effect on both the $J$-resistance curve and the overall ductility of the specimen, but relatively little effect on the maximum load. In contrast, variations due to strain hardening shown in Fig. 5 have a large effect on the maximum load but much less influence on the overall ductility. The $J$-resistance curve depends strongly on both $N$ and $f_{0}$. 

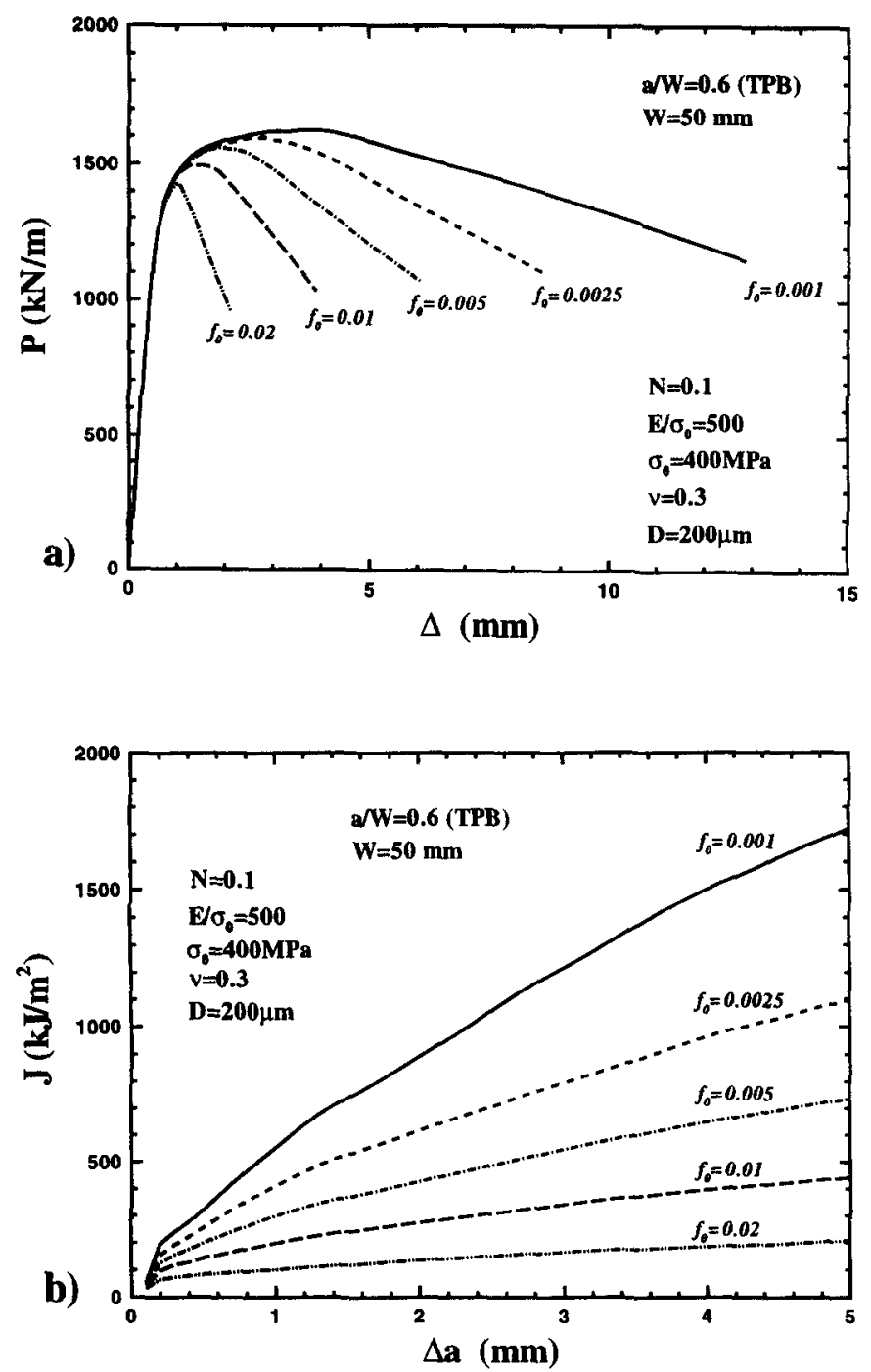

Fig. 6. Effect of variations in $f_{0}$ on the load-displacement relation in (a) and on the relation between $J$ and crack advance in (b). Three point bend geometry with $a / W=0.6$ and $W^{\prime}=50 \mathrm{~mm}$.

\section{THEORETICAL PREDICTIONS AND EXPERIMENTAL MEASUREMENTS FOR TWO STEELS AND FOUR SPECIMEN GEOMETRIES}

\subsection{A533B Steel}

Joyce and Link (1994) have obtained load-displacement and $J$-resistance curve data for A533B pressure vessel steel using all of the specimen geometries shown in Fig. 1 except the center cracked panel geometry. The inplane geometric parameters characterizing the specimens are shown in Fig. 1; the thickness of the specimens is 
denoted by $B$. All specimens tested had side grooves cut to a depth of $10 \%$ of the thickness on each lateral face, such that the ligament on the plane ahead of the crack had thickness $0.8 B$. The true stress-logarithmic strain curve (1) was fit to the tensile data for the steel using

$$
E=200 \mathrm{GPa}, \quad \sigma_{0}=400 \mathrm{MPa}, \quad N=0.1, \quad v=0.3 .
$$

The fit was made over the strain range starting at the $0.2 \%$ offset yield stress at 397 MPa to the true strain at maximum load, $\varepsilon=0.125$, corresponding to a true stress of $628 \mathrm{MPa}$. The true stress at $\varepsilon=0.125$ given by (1) with use of (7) is $605 \mathrm{MPa}$.

Once a sub-element scheme is fixed, several options could be adopted for choosing the two parameters $D$ and $f_{0}$. Here, Scheme A has been selected, and the two parameters have been chosen to accurately reproduce the experimentally measured resistance curves of $J$ vs $\Delta a$ shown on the left in the second plot from the top in Fig. 7(b). These data were obtained by Joyce and Link using deeply cracked, three point bend specimens (TPB) with $a / W=0.6, W=5.08 \mathrm{~cm}(2 \mathrm{in})$ and $B=2.54 \mathrm{~cm}(1 \mathrm{in})$. The TPB specimen generated the greatest amount of crack advance $(5 \mathrm{~mm})$, and this was the primary reason for selecting these data for the calibration. $J$-values were generated from the experimental records of load vs load-line displacement, $\Delta$, crack mouth opening displacement, $\Delta_{\mathrm{M}}$, and crack advance, $\Delta a$, using the ASTM procedure cited above. Experimental data from three nominally identical TPB specimens were obtained and are displayed in Fig. 7. The load per unit thickness, $P$, in the plots of experimental data is evaluated by dividing the total load by the reduced ligament thickness, $0.8 B$. The predicted curve of $J$ vs $\Delta a$ from the model for the TPB specimen is shown next to the experimental data on the right in Fig. 7(b) for the choice

$$
f_{0}=0.005 \text { and } D=200 \mu \mathrm{m} \text {. }
$$

(There is essentially no difference between the value of $J$ computed from the integral definition using a remote contour and that computed using the ASTM definition for the TPB geometry, as will be seen in Section 5.) All other model predictions displayed in Figs 7-10 for the A533B steel were computed using the material parameter values specified in (7) and (8). An alternative procedure for identifying $D$ and $f_{0}$ might make use of experimental data for load vs load-line displacement or, perhaps, for load vs crack mouth opening displacement, rather than $J$ vs $\Delta a$. However, the above choice reproduces these other two experimental records almost equally well, as is evident in Fig. 7(a). The largest discrepancy (as much as 10\%) between the computed curve for $P$ and the experimental one occurs in the initial stages of crack growth. It is likely that a more accurate representation than (1) of the actual tensile stress-strain data might improve agreement, but no further effort in this direction was undertaken in the present study. Also, as already indicated, it may be worthwhile to investigate sensitivity to the method of choosing $D$ and $f_{0}$ in subsequent work and to explore the effect of including a parameter to characterize delayed void nucleation.

The experimental records for the other three specimen geometries and loadings (CT, SENT and DENT) are shown on the left in each of the two parts of Fig. 7. The corresponding predictions computed using the model are shown to the right of the experimental data. The theoretical $J$-values were all computed using the line integral definition on a contour remote from the crack tip. It is evident from the plots of load 
Ductile crack growth under large scale yielding
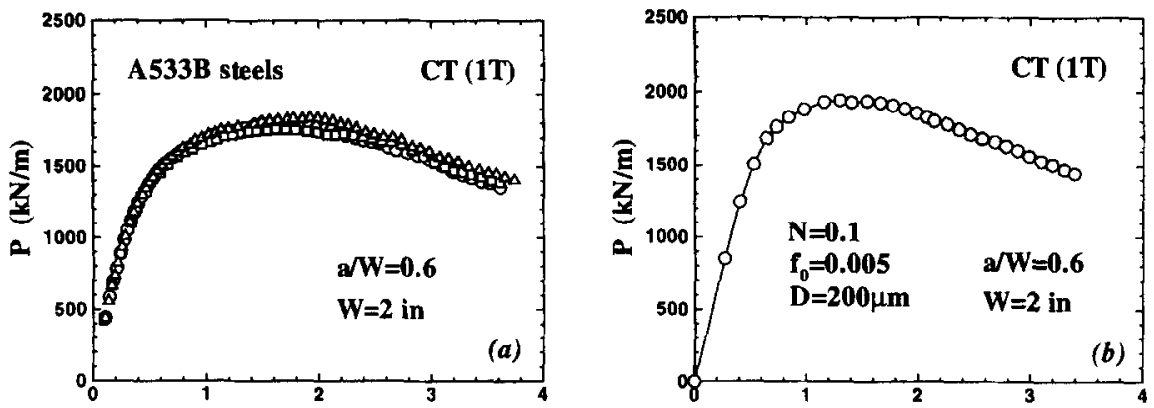

$\Delta(\mathbf{m m})$

$\Delta(\mathbf{m m})$
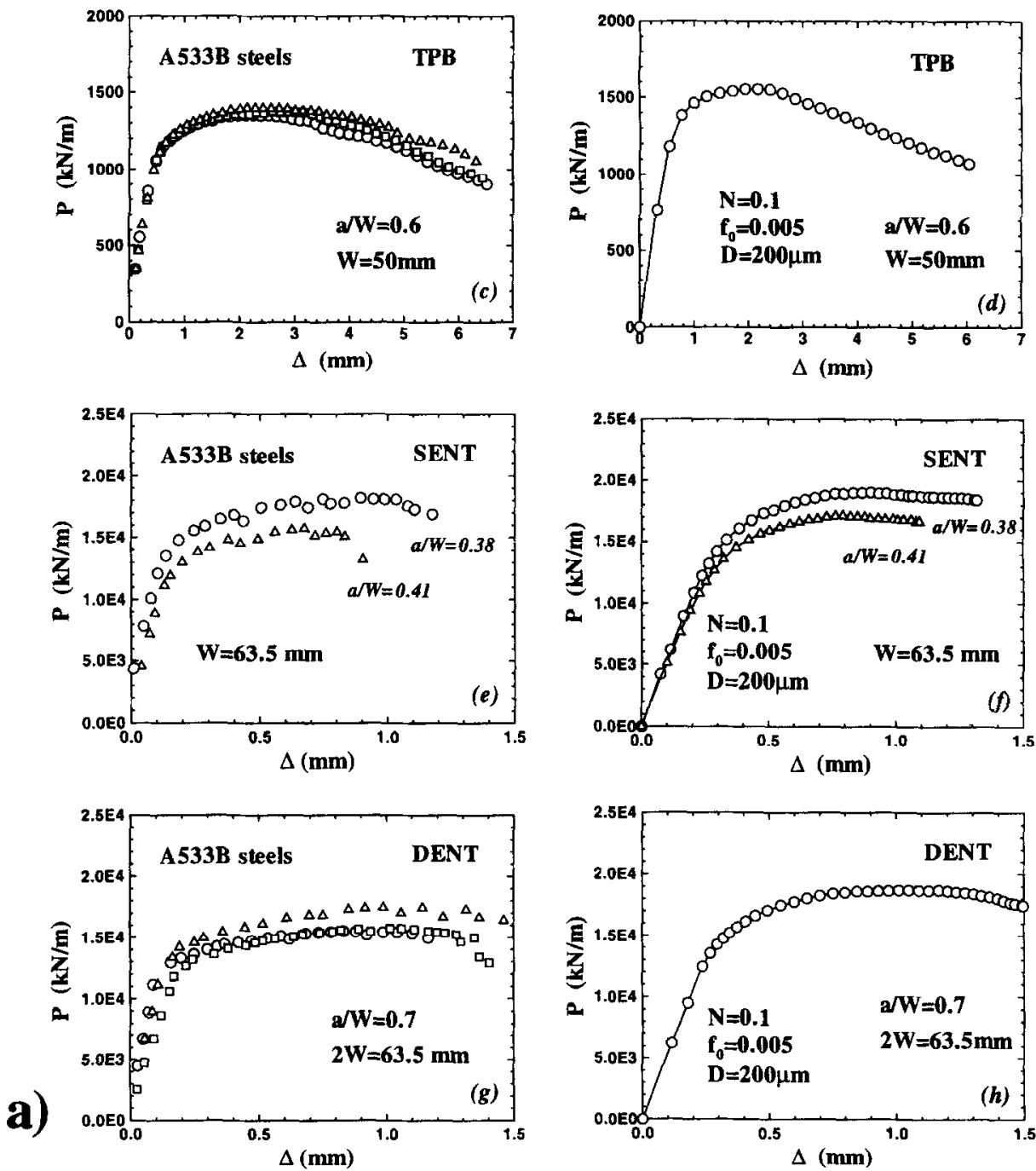

Fig. 7. Comparisons of experimental data for A533B steel (Joyce and Link, 1994) with predictions from the model for four types of specimens. The tensile stress-strain properties are given by $(7)$, and the parameters $D$ and $f_{0}$ in the model (8) have been chosen to reproduce the experimental curve of $J$ vs crack advance $\Delta a$ for the TPB specimen, as discussed in the text. (a) Load per unit thickness, $P$, vs load-line displacement, $\Delta$; (b) $J$ vs $\Delta a$. In each plot, the experimental data are on the left and the theoretical predictions are on the right. 

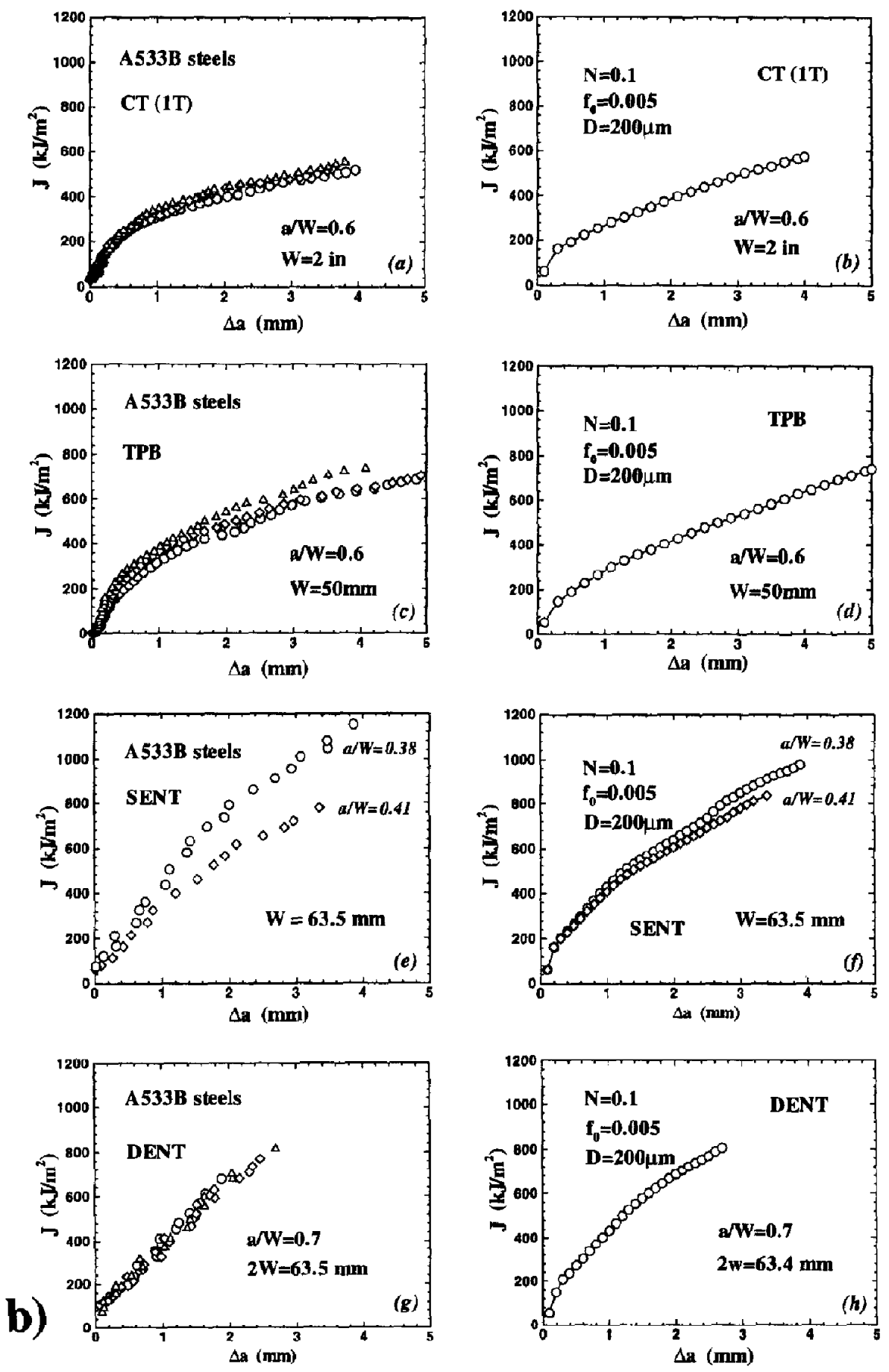

Fig. 7.-Continued 

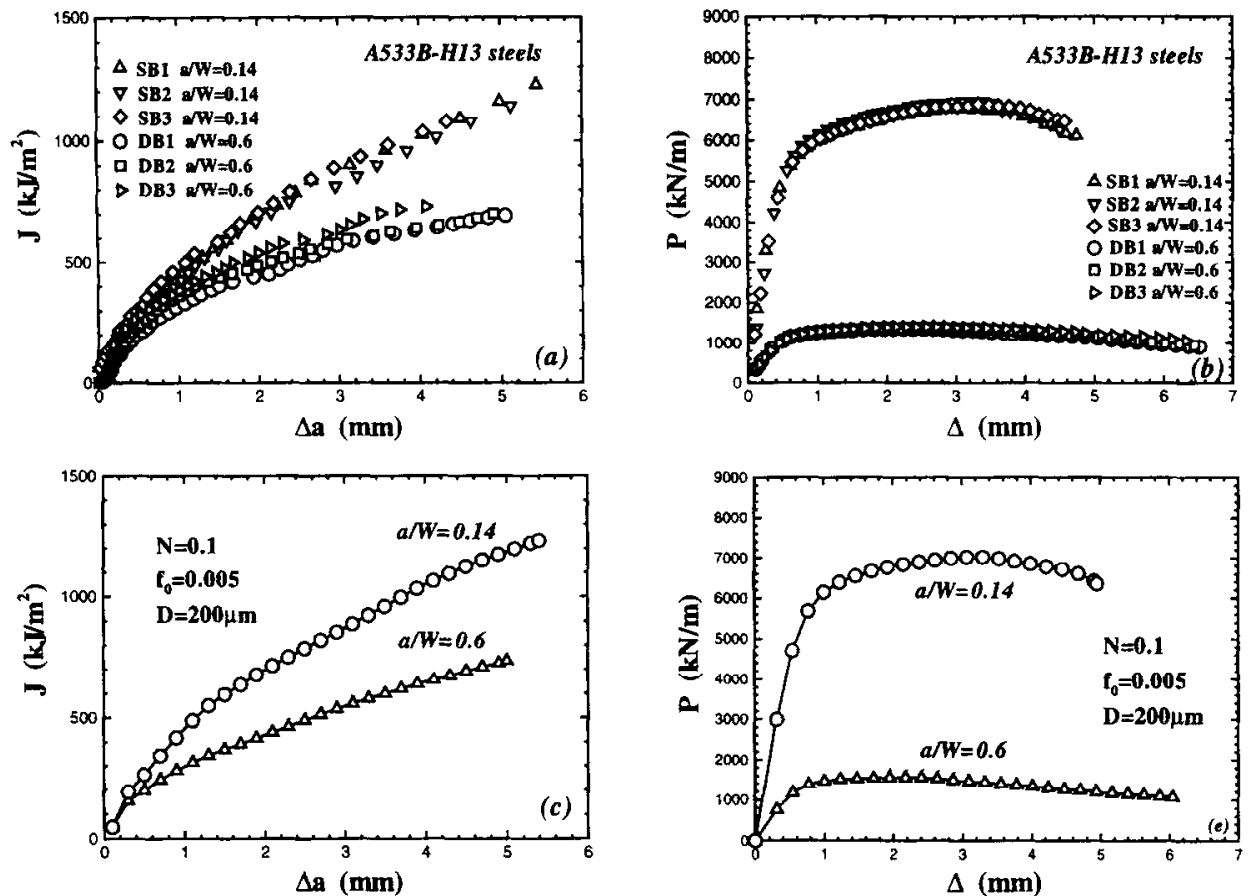

Fig. 8. Effect of two widely different ratios, $a / W$, of initial crack length to specimen width for three point bend specimens (TPB) of A533B steel. Experimental data (Joyce and Link, 1994) for three nominally identical specimens at for each value of $a / W$ are shown in the top plots with the model predictions in the lower plots. $W=5.08 \mathrm{~cm}(2 \mathrm{in})$.

vs load-line displacement $\Delta$ that crack growth occurs in these specimens under large scale yielding conditions. There is considerable variation in behavior over the four types of specimens, and, for the most part, the model predictions quantitatively reproduce these differences. In particular, the significant differences among the $J$ resistance curves are fairly accurately captured by the model. These differences have been attributed to the differing conditions of triaxial constraint from one type of specimen to the other, as discussed most recently by Hancock et al. (1993). The voids in the elements ahead of the tip in the computational model grow at a rate which depends on the level of triaxial stress, thereby incorporating this important effect on the fracture process.

The effect of a small change in the ratio $a / W$, from 0.38 to 0.41 , can be seen for the SENT specimen in Fig. 7. The effect of a large change in $a / W$, from a deep crack to a relatively shallow crack, is displayed for the three point bend geometry in Fig. 8. The experimental data and the model predictions for the deep crack, $a / W=0.6$, are the same as those shown in Fig. 7 for the TPB geometry. Recall that it was this set of data which was used to fix $D$ and $f_{0}$. The significantly higher load carrying capacity of the specimen with the shallow crack is accurately predicted by the model. The relatively smaller, but significant, geometry dependence of the $J$-resistance curve is also reproduced. The resistance curve of the shallow-cracked specimen departs from that of the deeply-cracked specimen after less than a millimeter of crack growth. 


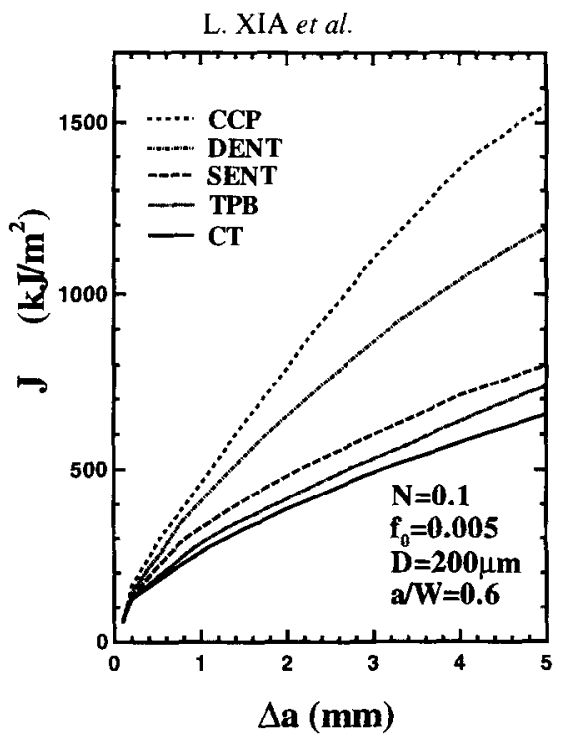

Fig. 9. Curves of $J$ vs $\Delta a$ as predicted by the model for A533B for the five geometry and loading combinations of Fig. 1. In each case here, the material parameters of the model are specified by (7) and (8), with $a / W=0.6$ and $W=5.08 \mathrm{~cm}(2 \mathrm{in})$.

A summary plot which emphasizes the geometry dependence of the $J$-resistance behavior for the A533B steel is shown in Fig. 9. The model predictions for each of the five geometries shown in Fig. 1 are plotted together, all for the case $a / W=0.6$ corresponding to a relatively deep crack. The $J$-values in these plots were computed using the line integral on a contour remote from the tip. The trends follow those brought out in earlier modeling work by Sun and Schmitt (1990) and in the systematic experimental studies of the effect of constraint by Hancock et al. (1993) and Joyce and Link (1994). The deeply-cracked CT and TPB specimens have the highest constraint and lowest resistance curves, while the center-cracked panel (CCP) has the lowest constraint and the highest resistance curve.

One feature evident in Fig. 9, and in Figs 7 and 8 as well, is the fact that there appears to be little geometry dependence at initiation. While the value of $J$ at initiation is somewhat sensitive to its practical definition, recent experimental studies such as those of Hancock et al. (1993) and Joyce and Link (1994) on several tough steels have detailed the weak geometric dependence of $J$-resistance behavior at initiation. This feature emerges most clearly from the embedded fracture process model of Tvergaard and Hutchinson (1994). According to that model, crack growth begins when $J$ attains the work of the fracture process, $\Gamma_{0}$, independent of the geometry. As discussed in Section 2 , for the ductile process under consideration here, $\Gamma_{0} \cong \sigma_{0} D / 2$, which from (7) and (8) is approximately $40 \mathrm{kJm}^{-2}$ for A533B. This value is generally in accord with the levels of $J$ in the earliest stage of growth for all the results shown in Figs 7-9.

There is a striking difference between the $J$-resistance curves under large scale yielding conditions such as those in Fig. 9 and the small scale yielding resistance curve in Fig. 4 for the same set of material parameters $\left(f_{0}=0.005\right.$ and $\left.D=200 \mu \mathrm{m}\right)$. The curve in Fig. 4 is presented using dimensionless variables, but it is nevertheless evident that the resistance has nearly reached steady-state conditions (i.e. $\mathrm{d} J / \mathrm{d} a=0$ ) for 


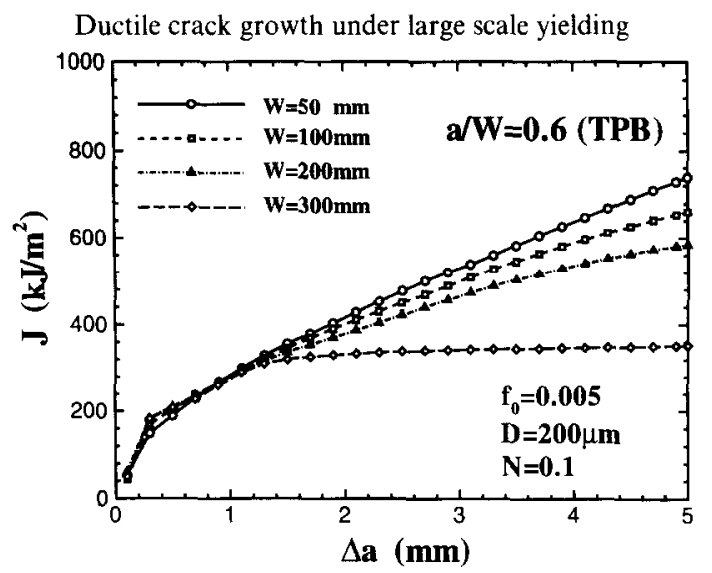

Fig. 10. The effect of specimen size on crack growth resistance curves as predicted for the three point bend specimen with $a / W=0.6$ for material parameters chosen to reproduce the behavior of $\mathrm{A} 533 \mathrm{~B}$. The specimen with $W=300 \mathrm{~mm}$ corresponds to small scale yielding conditions.

$\Delta a=10 D$ corresponding to $2 \mathrm{~mm}$ of crack growth. The large scale yielding resistance curves in Fig. 9 all have an appreciable slope at $\Delta a=2 \mathrm{~mm}$ and are not yet close to an asymptotic limit at $5 \mathrm{~mm}$ of crack advance. The trend from large scale yielding behavior to small scale yielding behavior is shown in Fig. 10 for the three point bend specimen with $a / W=0.6$. Numerically calculated curves of $J$ vs $\Delta a$ are presented for four sizes of specimens, $W=5,10,20$ and $30 \mathrm{~cm}$. The curve for $W=5 \mathrm{~cm}$ is the same as that for the TPB specimen in Fig. 9, while that for the largest specimen, $W=30$ $\mathrm{cm}$, essentially coincides with the small scale yielding resistance curve in Fig. 4. Calculations were carried out for even larger sizes, but these coincided with those shown for $W=30 \mathrm{~cm}$. For less than about $1.5 \mathrm{~mm}$ of crack growth, there is little dependence on specimen size. However, for larger amounts of growth, the larger specimens display significantly less crack growth resistance than the smaller specimens. A dramatic drop in the slope of the crack growth resistance curve occurs for $\Delta a$ greater than about $2 \mathrm{~mm}$ for specimens large enough such that small scale yielding conditions are approached. This trend has serious implications for efforts to use $J$ resistance curve data obtained from small specimens under large scale yielding conditions in applications to cracks in thick sections where the behavior will be closer to small scale yiclding.

\subsection{A710 Steel}

Joyce and Hackett (1991) carried out a limited experimental program on the steel A710, which is both stronger and tougher than A533B. Here attention is directed to the two sizes of compact tension specimen (CT) which were tested, each with $a / W=0.6$ and each with a total reduced ligament of $0.8 B$ due to side grooves. Two specimens, designated by $1 T$, had $B=2.54 \mathrm{~cm}(1 \mathrm{in})$ and $W=5.08 \mathrm{~cm}(2 \mathrm{in})$, and two, designated by $2 T$, had $B=5.08 \mathrm{~cm}$ ( 2 in) and $W=10.16 \mathrm{~cm}(4 \mathrm{in})$. The experimental data obtained from these four specimens are shown in the top plots in Fig. 11. The load per unit thickness, $P$, is again taken as the total load divided by the reduced thickness $0.8 B$. For both experimental data and the model predictions, there 

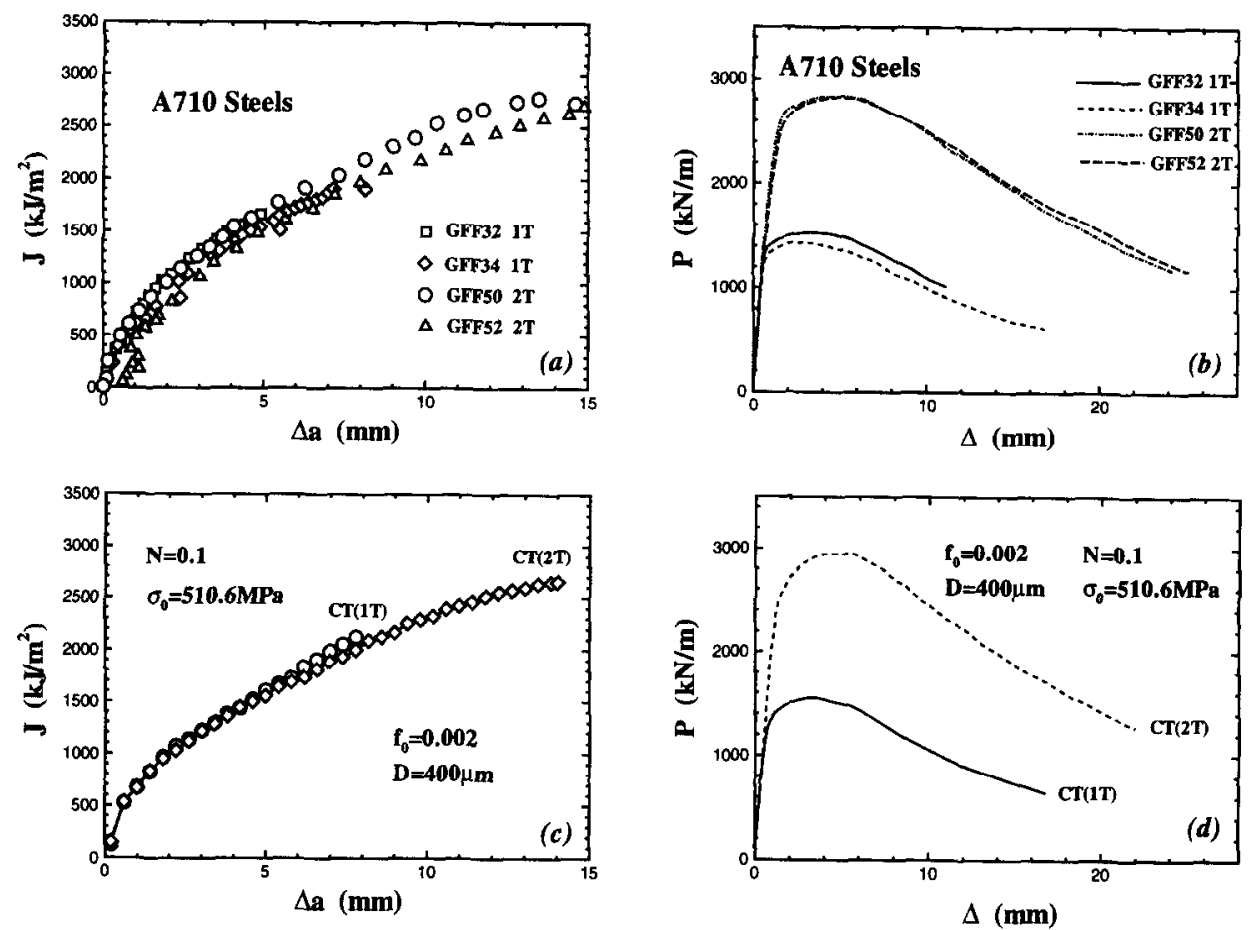

Fig. 11. The effect of specimen size on the behavior of compact tension (CT) specimens of A710 steel with $a / W=0.6$. Experimental data of Joyce and Hackett (1991) are shown in the upper plots, with the model predictions shown below. The specimens designated by $1 T$ have $B=2.54 \mathrm{~cm}$ ( 1 in) and $W=5.08 \mathrm{~cm}$ ( 2 in). The specimens designated by $2 T$ are twice as large in all dimensions. Data for $1 T$ were used to choose $D$ and $f_{0}$.

is essentially no difference between the load-line displacement, $\Delta$, and the crack mouth opening displacement at the load line.

Computations based on the model were again carried out using sub-element Scheme A. Tensile stress-strain data for the A710 were fit using $E=210 \mathrm{GPa}, \sigma_{0}=510.6$ $\mathrm{MPa}, N=0.1$ and $v=0.3$. Values of $f_{0}$ and $D$ were chosen to reproduce the curve of $J$ vs $\Delta a$ for the $1 T$ specimens in Fig. 11 , giving $f_{0}=0.002$ and $D=400 \mu \mathrm{m}$. The $J$-values were computed on a remote contour from the tip using the line integral definition. The full set of computed results for both sizes of specimen is shown in the lower plots in Fig. 11. The size effect itself is fairly small in this instance, as noted from the near-invariance of the data for $J$ vs $\Delta a$ and from the approximate scaling of the curve of $P$ vs $\Delta$ with size. Specimen thickness, $B$, is not a variable in the model results since the calculations are carried out in plane strain. A thickness dependence does not seem to be present in the normalized experimental data. The model is remarkably successful in reproducing both $J$-resistance behavior and load-displacement behavior. For the $2 T$ specimen, the model faithfully replicates the experimental data for crack advances of almost $15 \mathrm{~mm}$, corresponding to $J$-values which increase to more than 25 times the initiation value $\left(\approx 100 \mathrm{kJm}^{-2}\right)$. 


\section{CONCLUDING REMARKS AND SUGGESTIONS FOR FURTHER WORK}

The procedure laid out for applying the model involves selection of the parameters $D$ and $f_{0}$ such that the model reproduces one set of experimental crack growth data for that material. The data could be in the form of a $J$-resistance curve or, in principle, it could be data for load vs load-line displacement. Since there may be some dependence on the sub-element scheme chosen to represent the slab of void-containing elements, the scheme should be chosen prior to selection of $D$ and $f_{0}$ and fixed thereafter. In this paper, it has been demonstrated that the model can be used in this way to predict accurately the most important aspects of behavior for specimen geometries and loadings chosen to span the widest possible range of crack tip triaxiality for two tough steels under conditions of fully plastic yielding. Nevertheless, it is likely that further work will be needed to validate the model. Further effort may be needed to establish the best way to select the two damage parameters, $D$ and $f_{0}$, and to gauge the sensitivity of model predictions to these parameters. In particular, it will be of interest to assess the performance of the model against some alloys of intermediate toughness and tearing resistance. The fracture behavior of specimens or components of the steels considered here is relatively insensitive to conditions surrounding the initiation of crack growth because of the high tearing resistance of these steels. Since there is some sensitivity in the early stages of crack growth to the choice of sub-element scheme, it will be important to understand how this affects predictions for material alloys with less tearing resistance than those considered here. If greater accuracy than that demonstrated here is required of the method, especially in the early stages of crack growth, then it is likely that effort will be needed to study the influence of a more accurate representation of the actual tensile stress-strain behavior and, possibly, on the inclusion of additional damage parameters such as one to characterize void nucleation.

Although the parameters $D$ and $f_{0}$ are micro-structurally based, they are used here in a phenomenological manner in that they are chosen to reproduce test data from a cracked specimen and not the actual void spacing and initial volume fraction. To the extent that these parameters represent the actual microstructure, the model should be useful for gaining a better understanding of the connection between microstructure and crack growth resistance.

The model can be employed to check the utility of other measures of crack tip behavior for growing cracks than those discussed above. One such measure is $J_{\mathrm{M}}$ suggested by Ernst (1989). Ernst's measure, which is limited to deeply-cracked specimens, is defined in terms of data for load vs load-line displacement and crack advance. Figure 12 gives plots of three measures, denoted by $J_{\text {path }}, J_{\eta}$ and $J_{\mathrm{M}}$, as a function of $\Delta a$ for both the three point bend specimen and the compact tension specimen, in each case for $a / W=0.6$ and with the other parameters specified in the figure. Here, $J_{\text {path }}$ is the notation for the value of $J$ computed from the line integral definition using a contour remote from the tip, $J_{\eta}$ is the ASTM definition referred to in Section 2, and $J_{M}$ is computed using the recipe of Ernst. These curves have been calculated using the respective definitions of the $J$-quantities from the predictions of the model with subelement Scheme A. Figure 12 displays the tendency of $J_{\mathrm{M}}$ to drift above the other two 

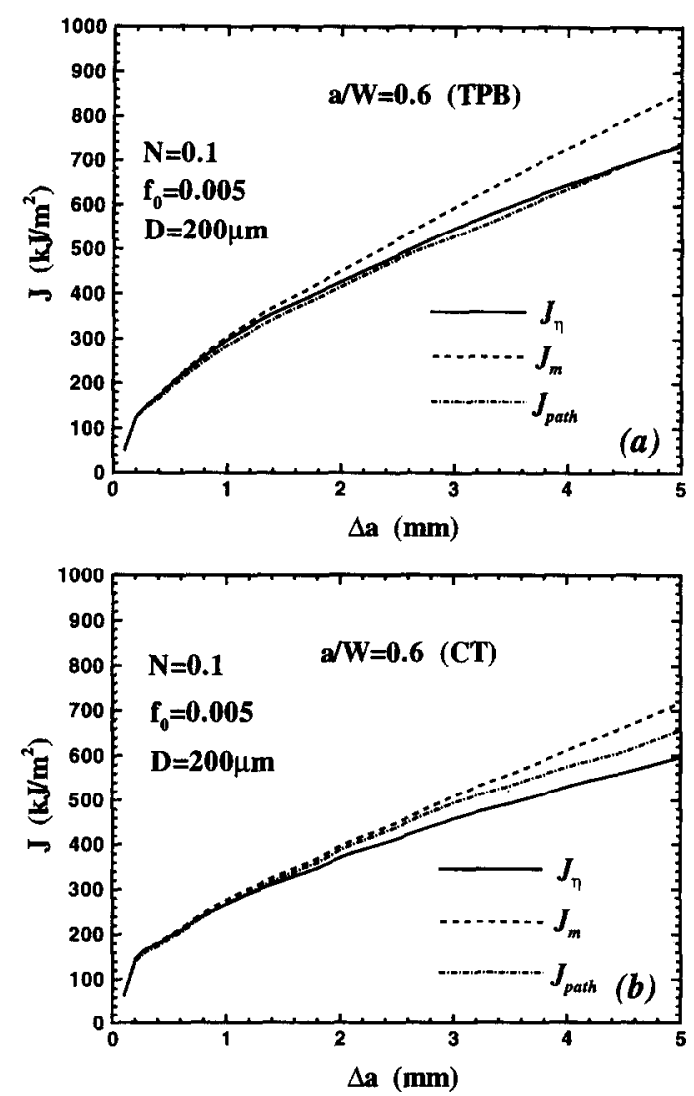

Fig. 12. Curves of $J$ vs $\Delta a$ for (a) a deeply-cracked three point bend (TPB) specimen and (b) for a deeplycracked compact tension (CT) specimen. Here, $J_{\text {path }}$ denotes the value computed by line integral on contours remote from the tip, $J_{\eta}$ denotes the measure proposed by ASTM, and $J_{\mathrm{M}}$ denotes the measure proposed by Ernst. The latter two measures are generated from the computed records of load and crack advance vs load-line displacement.

measures as the crack advances. The compact tension specimen also shows some divergence between $J_{\text {path }}$ and $J_{\eta}$ for crack advance exceeding several millimeters, although not more than $10 \%$.

The tendency for $J_{\mathrm{M}}$ to lie above the other two measures has been noted in earlier efforts to analyze experimental crack growth data in tough steels, leading in some instances to significant divergence with curves of $J_{\mathrm{M}}$ versus $\Delta a$ having slopes which increase as $\Delta a$ increases (Joyce et al., 1990). In other calculations, not displayed here, we have also found numerical examples based on the present model where the resistance curve based on $J_{\mathrm{M}}$ has a slope that increases with crack advance after an initial growth phase, while the curves based on the other two measures have monotonically decreasing slopes. Although such trends would seem to be unrealistic, a divergence between $J_{\mathrm{M}}$ and the other two measures is not necessarily grounds for concluding that $J_{\mathrm{M}}$ has less validity than the other measures. Any real test of that question would require an assessment of whether $J_{\mathrm{M}}$ could be used reliably to predict load-displacement behavior and crack stability, given resistance curve data expressed 
in terms of $J_{\mathrm{M}}$. Such a test would necessarily require that the level of stress triaxiality be more or less constant, since it is evident that no approach can be based on a single parameter resistance curve. The present computational model could be used to explore such issues. It does seem clear, however, that one would invite error if one were to "mix" $J$-measures. For example, unconservative predictions would almost certainly ensue if one used $J_{\mathrm{M}}$ to generate resistance curve data and then used those data expressed in terms of one of the other measures to predict load-displacement response of a structural component. In this connection, it is worth mentioning that $J_{\mathrm{M}}$ has only been defined for deeply-cracked geometries, and it is not clear how to apply this measure to most structural applications. As emphasized in the Introduction, one of the main advantages of the present computational model, or of any of the other models of its type, is the fact that the resistance curve is relegated to a secondary role. Indeed, one can use the model to predict structural response under conditions of crack advance without recourse to any resistance curve.

Extensions of the model to cope with important situations where the crack advances out of its plane are possible but will require considerable additional work. The effect of shear localization, which becomes important in some of the very high strength steels, will be particularly challenging. One important application which does seem feasible for investigation without major innovation is the three-dimensional problem of an elliptical surface crack in a thick plate undergoing either bending or stretching. This problem would be a good test for the model because stress triaxiality varies along the crack front, with the highest triaxiality at the interior and the lowest where the crack front intersects the surface.

\section{ACKNOWLEDGEMENTS}

The work of LX and CFS was supported by Grant N00167-92-K-0038 from the David Taylor Research and Development Center and from Battelle Research Labs, Columbus, $\mathrm{OH}$, funded by the Nuclear Regulatory Commission. The work of JWH was supported in part by the National Science Foundation from Grant MSS-92-02141 and in part by the Division of Applied Sciences, Harvard University.

\section{REFERENCES}

ASTM (1993) E 1152-87 Standard test method for determining $J-R$ curves. Annual Book of ASTM Standards. American Society of Testing Materials, Philadelphia, PA.

Becker, R., Needleman, A., Suresh, S., Tvergaard, V. and Vasudevan, A. K. (1989) An analysis of ductile failure by grain boundary void growth. Acta Metall. 37, 99-120.

Bethmont, M., Rousselier, G., Kussmaul, K., Sauter, A. and Jovanovic, A. (1990) The local approach of fracture and its application to a thermal shock experiment. Nucl. Engng Design 119, 249-261.

Bilby, B. A., Howard, I. C. and Li, Z. H. (1993) Prediction of the first spinning cylinder test using ductile damage theory. Fatigue Fract. Engng Mater. Struct. 16, 1-20.

Brocks, W., Klingbeil, Kunecke, D. and Sun, D.-Z. (1995) Application of the Gurson model to ductile tearing resistance. Constraint Effects in Fracture: Theory and Applications, ASTM STP 1244 (ed. M. Kirk and A. Bakker). American Society for Testing Materials, Philadelphia, PA. 
Budiansky, B. and Summer, E. E. (1985) On size effects in plane stress crack-growth resistance. Developments in Mechanics, Vol. 13, Proceedings of the 19th Midwestern Mechanics Conference, Ohio State University, Columbus, OH, 9-11 Sept, 1985.

Drugan, W. J., Rice, J. R. and Sham, T.-L. (1982) Asymptotic analysis of growing plane strain tensile cracks in elastic -ideally plastic solids. J. Mech. Phys. Solids 30, 447-473.

Ernst, H. A. (1989) Further developments on the modified $J$-integral. Nonlinear Fracture Mechanics: Volume 11-Elastic-Plastic Fracture, ASTM STP 995 (ed. J. D. Landes, A. Saxena and J. G. Merkle), pp. 306-319. American Society of Testing Materials, Philadelphia, PA.

Gurson, A. L. (1977) Continuum theory of ductile rupture by void nucleation and growth: Part I-yield criteria and flow rules for porous ductile media. J. Engng Mater. Technol. 99, 2-15.

Hancock, J. W., Reuter, W. G. and Parks, D. M. (1993) Constraint and toughness parameterized by T. Constraint Effects in Fracture, ASTM STP 1171 (ed. E. M. Hackett, K. H. Schwalbe and R. H. Dobbs), pp. 21-40. American Society for Testing Materials, Philadelphia, PA.

Hellman, D. and Schwalbe, K. H. (1984) Geometry and size effects on $J-R$ and $\Delta-R$ curves under plane stress conditions. Fracture Mechanics: Fifteenth Symposium, ASTM STP 883 (ed. A. R. Sanford), pp. 577-605. American Society of Testing Materials, Philadelphia, PA.

Hermann, L. and Rice, J. R. (1980) Comparison of theory and experiment for elastic-plastic plane strain crack growth. Metal Sci. 14, 285-291.

Joyce, J. A., Davis, D. A., Hackett, E. M. and Hays, R. A. (1990) Application of the $J$-integral and the modified $J$-integral to cases of large crack extension. Fracture Mechanics: Twenty First Symposium, ASTM STP 1074 (ed. J. P. Gudas, J. A. Joyce and E. M. Hackett), pp. 85105. American Society of Testing Materials, Philadelphia, PA.

Joyce, J. A. and Hackett, E. M. (1991) Development of an engineering definition of the extent of $J$-controlled crack growth. Defect Assessment of Components-Fundamentals and Applications, ESIS/EGF9 (ed. J. G. Blaud and K. H. Schwalbe), pp. 233-249. Mechanical Engineering Publications, London.

Joyce, J. A. and Link, R. E. (1994) Effects of constraint on upper shelf fracture toughness. Fracture Mechanics: 26th Volume, ASTM STP 1256 (ed. W. G. Reuter, J. H. Underwood and J. C. Newman, Jr). American Society for Testing Materials, Philadelphia, PA.

Kanninen, M. F., Rybicki, E. F., Stonesifer, R. B., Broek, D., Rosenfield, A., Marshall, C. W. and Hahn, G. T. (1979) Elastic-plastic fracture mechanics for two dimensional stable crack growth and instability problems. Elastic-Plastic Fracture, ASTM STP 668 (ed. J. D. Landes, J. A. Begley and G. A. Clarke), pp. 121-150. American Society for Testing Materials, Philadelphia, PA.

Li, Z. H., Bilby, B. A. and Howard, I. C. (1993) A study of the internal parameters of ductile damage theory. Fatigue Fract. Engng Mater. Struct. (to be published).

McClintock, F. A. (1971) Plasticity aspects of fracture. In Fracture, Vol. 3 (ed. H. Liebowitz), pp. 47-725. Academic Press, New York

McMeeking, R. M. (1977) Finite deformation analysis of crack-tip opening in elastic-plastic materials and implications for fracture. J. Mech. Phys. Solids 25, 357-391.

Mudry, F., di Rienzo, F. and Pineau, A. (1989) Numerical comparison of global and local fracture criteria in CT and CCP specimens. Nonlinear Fracture Mechanics: Volume 11-Elastic-Plastic Fracture, ASTM STP 995 (ed. J. D. Landes, A. Saxena and J. G. Merkle), pp. 24-39. American Society of Testing Materials, Philadelphia, PA.

Needleman, A. and Tvergaard, V. (1987) An analysis of ductile rupture modes at a crack tip. J. Mech. Phys. Solids 35, 151-183.

Newman, J. C., Dawicke, D. S., Sutton, M. A. and Bigelow, C. A. (1993) A fracture criterion for widespread cracking in thin-sheet aluminum alloys. Proc. 17th Symp. Int. Committee on Aeronautical Fatigue, 9-11 June 1993, Stockholm.

Rousselier, G. (1987) Ductile fracture models and their potential in local approach of fracture. Nucl. Engng Design 105, 97-111.

Rousselier, G., Devaux, J.-C., Mottet, G. and Devesa, G. (1989a) A methodology for ductile 
fracture analysis based on damage mechanics : an illustration of a local approach of fracture. Nonlinear Fracture Mechanics: Volume 11-Elastic-Plastic Fracture, ASTM STP 995 (ed. J. D. Landes, A. Saxena and J. G. Merkle), pp. 332-354. American Society of Testing Materials, Philadelphia, PA.

Rousselier, G., Devesa, G. and Bethmont, M. (1989b) Effect of specimen geometry on $J$ resistance curves in near small scale yielding conditions. 7 th Int. Conf. on Fracture, Houston, TX.

Shih, C. F., deLorenzi, H. G. and Andrews, W. R. (1979) Studies on crack initiation and stable crack growth. Elastic-Plastic Fracture, ASTM STP 668 (ed. J. D. Landes, J. A. Begley and G. A. Clarke), pp. 65-120. American Society for Testing Materials, Philadelphia, PA.

Shih, C. F. and Xia, L. (1995) Modeling crack growth resistance using computational cells with microstructurally-based length scales. Constraint Effects in Fracture: Theory and Applications, ASTM STP 1244 (ed. M. Kirk and A. Bakker). American Society for Testing Materials, Philadelphia, PA.

Sun D.-Z. and Schmitt, W. (1990) Application of micromechanical models to the analysis of ductile fracture resistance behaviour. Numerical Methods in Fracture Mechanics, Proceedings of the 5th Int. Conf. held in Freiburg, FRG, 23-27 April, 1990 (ed. A. R. Luxmoore and D. R. J. Owen), pp. 275-286.

Tvergaard, V. (1982) Influence of void nucleation on ductile shear fracture at a free surface. $J$. Mech. Phys. Solids 30, 399-425.

Tvergaard, V. (1990) Material failure by void growth to coalescence. Advances in Applied Mechanics, Vol 27 (ed. J. W. Hutchinson and T. Wu), pp. 83-151. Academic Press, New York.

Tvergaard, V. and Hutchinson, J. W. (1992) The relation between crack growth resistance and fracture process parameters in elastic-plastic solids. J. Mech. Phys. Solids 40, 1377-1397.

Tvergaard, V. and Hutchinson, J. W. (1994) Effect of $T$-stress on mode I crack growth resistance in a ductile solid. Int. J. Solids Struct. 31, 823-833.

Tvergaard, V. and Needleman, A. (1984) Analysis of the cup-cone fracture in a round tensile bar. Acta Metall. 32, 157-169.

Xia, L. and Shih, C. F. (1995) Ductile crack growth-I. A numerical study using computational cells with microstructurally-based length scales. J. Mech. Phys. Solids 43, 233-259. 